\title{
Chronic exposure to tumor necrosis factor alpha induces retinal pigment epithelium cell dedifferentiation
}

\author{
Sara Touhami ${ }^{1,2^{*}} \mathbb{D}$, Fanny Beguier ${ }^{1}$, Sébastien Augustin ${ }^{1}$, Hugo Charles-Messance ${ }^{1}$, Lucile Vignaud ${ }^{1}$, \\ Emeline F. Nandrot ${ }^{1}$, Sacha Reichman ${ }^{1}$, Valérie Forster ${ }^{1}$, Thibaud Mathis ${ }^{1}$, José-Alain Sahel ${ }^{1,3}$, Bahram Bodaghi ${ }^{2}$, \\ Xavier Guillonneau ${ }^{1}$ and Florian Sennlaub ${ }^{1}$
}

\begin{abstract}
Background: The retinal pigment epithelium (RPE) is a monolayer of pigmented cells with important barrier and immuno-suppressive functions in the eye. We have previously shown that acute stimulation of RPE cells by tumor necrosis factor alpha (TNFa) downregulates the expression of OTX2 (Orthodenticle homeobox 2) and dependent RPE genes. We here investigated the long-term effects of TNFa on RPE cell morphology and key functions in vitro.

Methods: Primary porcine RPE cells were exposed to TNFa (at $0.8,4$, or $20 \mathrm{ng} / \mathrm{ml}$ per day) for 10 days. RPE cell morphology, phagocytosis, barrier- and immunosuppressive-functions were assessed.

Results: Chronic (10 days) exposure of primary RPE cells to TNFa increases RPE cell size and polynucleation, decreases visual cycle gene expression, impedes RPE tight-junction organization and transepithelial resistance, and decreases the immunosuppressive capacities of the RPE. TNFa-induced morphological- and transepithelial-resistance changes were prevented by concomitant Transforming Growth Factor $\beta$ inhibition.

Conclusions: Our results indicate that chronic TNFa-exposure is sufficient to alter RPE morphology and impede cardinal features that define the differentiated state of RPE cells with striking similarities to the alterations that are observed with age in neurodegenerative diseases such as age-related macular degeneration.
\end{abstract}

Keywords: Retinal pigment epithelium, Tumor necrosis factor alpha, Transforming growth factor beta, Age-related macular degeneration, Neuroinflammation, Neurodegenerative disease

\section{Background}

The retinal pigment epithelium (RPE) is a monolayer of polarized pigmented cells, located between the photoreceptor outer segments (POS) and the choroid. These highly differentiated cells play a crucial role in the visual cycle, the recycling of POS, the formation of the outer blood-retinal barrier [1], and in providing immunosuppressive signals that eliminate infiltrating immune cells from the subretinal space [2].

\footnotetext{
* Correspondence: saratouhami@gmail.com

'Sorbonne Université, INSERM, CNRS, Institut de la Vision, 17 rue Moreau, F-75012 Paris, France

${ }^{2}$ Department of Ophthalmology, Reference Center in Rare diseases, DHU Sight Restore, Hôpital Pitié Salpêtrière, University Paris Vl, 47-83 Boulevard de l'Hôpital, 75013 Paris, France

Full list of author information is available at the end of the article
}

With age, RPE cells decline in number and the percentage of multinucleated RPE cells increases [3, 4]. Enlarged, irregularly shaped, multinucleated RPE cells are also found in increased numbers in the vicinity of drusen that define early intermediate age-related macular degeneration (AMD) [5]. AMD is also associated with dysfunctional RPE: the visual cycle is slowed, evidenced by an increase of recovery time after bleach [6, 7]; the outer blood-retinal barrier is breached, illustrated by subretinal neovascularization, edema, and macrophages $(\mathrm{M} \phi)$ that accumulate subretinally around the RPE [2]. At later stages the RPE degenerates, forming atrophic zones that define geographic atrophy (GA), the untreatable late form of AMD. Although it seems evident that RPE dysfunction and death is a pivotal part of AMD pathogenesis, it is not yet clear what leads to and aggravates the decay. 
We and others previously showed that M $\phi s$ accumulate on the apical side of the RPE adjacent to the atrophic zones that define GA, around choroidal neovascularizations (CNV) and around large drusen in intermediate AMD [2, 8-12]. Using a co-culture model, we recently demonstrated that $\mathrm{M \phi} \phi$-derived tumor necrosis factor alpha (TNF $\alpha$ ) acutely downregulates the transcription factor orthodenticle homeobox 2 (OTX2) that is constitutively expressed in RPE cells, where it controls the expression of a number of essential genes. TNF $\alpha$ is a potent inflammatory cytokine, mainly produced by activated M $\phi$ s and T-cells, with a broad range of biological activities [13]. A possible role of TNF $\alpha$ in ageand AMD-related RPE changes is also suggested by the observation that plasmatic TNF $\alpha$ concentrations increase with age $[14,15]$ and are further increased in complement factor $\mathrm{H}$ (CFH) AMD-risk variant carriers [16]. Furthermore, TNF $\alpha$ expression in monocyte-derived M $\phi s$ increases with the age of the donor $[17,18]$ and AMD patients whose monocyte-derived M $\phi s$ express the greatest amount of TNF $\alpha$ have a higher prevalence of CNV [19].

We here show that chronic (10 days) exposure of primary RPE cells to TNF $\alpha$ increases RPE cell size and polynucleation, decreases visual cycle gene expression, impedes RPE tight-junction organization and transepithelial resistance, and decreases the immunosuppressive capacities of the RPE. Some, but not all, of these effects were due to TNF $\alpha$-induced transforming growth factor $ß$ expression.

Taken together our results indicate that chronic TNF $\alpha$ exposure is sufficient to alter RPE morphology and impede cardinal features that define the differentiated state of RPE cells with striking similarities to RPE alterations with age and dysfunctions observed in AMD.

\section{Methods}

\section{RPE cell-cultures}

Isolation of primary porcine RPE cells was conducted according to a previously described protocol [20]. This procedure adheres to the European initiative for restricting animal experimentation because not a single animal was killed for our experimentation specifically. Briefly, porcine eyes were purchased from a local slaughterhouse (Guy Harang, Houdan, France) in agreement with the local regulatory department and veterinarians and transported in CO2independent medium (Thermo Fisher Scientific) 2 or $3 \mathrm{~h}$ after enucleation. Eyes were dissected and cleaned off surrounding cunjunctiva, tenon's capsule, and muscles then immersed $(2 \times 5$ minutes) in an antiseptic solution (Pursept-A Xpress, Merz Hygiene $\mathrm{GmbH}$ ). The anterior segment of the bulb including the lens, vitreous gel, and the retina were then removed to isolate an eye-cup made of sclera, choroidal, and RPE cells. Each eye cup was washed twice with PBS (Thermo Fisher Scientific) and incubated with $0.25 \%$ trypsin-EDTA (Thermo Fisher Scientific) for
75 min at $37{ }^{\circ} \mathrm{C}$. Trypsin-detached RPE cells were pipetted off the choroid and resuspended in Dulbecco's modified Eagle's medium (DMEM, Thermo Fisher Scientific) supplemented with decomplemented $20 \%$ fetal calf serum (FCS, Thermo Fisher Scientific) and 1\% penicillin/streptomycin (PS) (final concentrations $100 \mathrm{U} / \mathrm{ml}$ and $100 \mu \mathrm{g} / \mathrm{ml}$ ) antibiotic mixture. Purified cells were seeded in 60-mm Petri dishes in DMEM-FCS20\%-PS1\% and incubated in a 5\% $\mathrm{CO} 2$ regulated atmosphere at $37{ }^{\circ} \mathrm{C}$. The culture DMEMFCS20\%-PS1\% medium was changed after $24 \mathrm{~h}$. When the cells reached a 70-80\% confluence state (usually at day 4), $0.05 \%$ trypsin-EDTA was added for $5 \mathrm{~min}$ at $37{ }^{\circ} \mathrm{C}$. The cells were then seeded on either 96-well culture plates (Corning) at the concentration of 75,000 cells/well or in $6.5 \mathrm{~mm}$ trans-well polyester filters $(0.4 \mu \mathrm{m}$ pore size, Corning, Kennebunk, ME, USA) at a concentration of 100,000 cells/well in DMEM-FCS20\%-PS1\%. Visual confluence was obtained after 2 weeks in culture at $37{ }^{\circ} \mathrm{C}, 5 \% \mathrm{CO} 2$, and cells were used immediately for TNF $\alpha$ treatment.

\section{Human blood monocyte isolation and supernatant preparation}

Human blood from healthy donors was collected for monocyte isolation after written informed consent in the Centre National d'Ophtalmologie des Quinze-Vingts (Paris). The protocol was approved by the Direction Générale pour la Recherche et l'Innovation of the Ministère de l'Enseignement et de la Recherche (Dossier n ${ }^{\circ} 14.007$ ) and by the Commission Nationale de l'Informatique et des Libertés (N/Ref.: IFP/MKE/AR144088). Briefly, peripheral blood mononuclear cells were obtained by Ficoll gradient centrifugation of fresh human blood, washed three times with PBS, and negatively sorted using the EasyStep Human Monocyte Enrichment Cocktail without CD16 Depletion kit (StemCell Technologies).

Lipopolysaccharide (LPS, O127:B8)-stimulated monocyte (Mo) supernatant was produced by a 2 -h stimulation of freshly prepared Mo, followed by a 24 culture of $100,000 \mathrm{Mo}$ in $0.1 \mathrm{ml}$ of fresh DMEM containing $1 \%$ penicillin/streptomycin (without LPS). The SN were then stored at $-80{ }^{\circ} \mathrm{C}$ until use in RPE cultures.

\section{Culture protocols and cell treatments RPE monoculture}

RPE cells were kept in culture until confluence then serum starved the day before starting supernatant (SN), TNF $\alpha$ and/or anti TGF $\beta$ treatments, in order to minimize the effects of serum on culture outcomes. In supernatant experiments, Mo SN was added daily for a maximum duration of 10 days. In TNF $\alpha$ experiments, recombinant TNF $\alpha$ (from $R \& D$ ) was added daily in 1\%FCS-1\%PS DMEM at various concentrations: $0.8,4$, or $20 \mathrm{ng} / \mathrm{ml}$ for 10 days. In inhibition experiments, antiTGF $\beta$ (Sigma, ref. SB505124, 500 nM) was added daily 
and simultaneously with TNF $\alpha$ (in $1 \% \mathrm{FCS}-1 \% \mathrm{PS}$ DMEM), for 10 days. At the end of culture, cells were washed twice with PBS and fixed in 4\% paraformaldehyde (PFA) for $10 \mathrm{~min}$. Alternatively, RA1 buffer was added to the cells to perform real-time quantitative polymerase chain reaction (RT-qPCR).

\section{RPE-monocyte coculture}

To evaluate the possible changes in their immunosuppressive capacity, RPE cells were pretreated during 10 days in 1\%FCS-1\%PS DMEM +/- different concentrations of recombinant TNF $\alpha$ before adding freshly purified human monocytes. For coculture purposes, RPE cells were serum and TNF $\alpha$ starved the day before adding 100,000 monocytes to RPE cells in each well. Cells were incubated at $37^{\circ} \mathrm{C}$ for a total of $24 \mathrm{~h}$.

\section{Immunofluorescence microscopy}

At the end of culture, cells were fixed in 4\% PFA for $10 \mathrm{~min}$ then washed twice in PBS and incubated for $2 \mathrm{~min}$ in a permeabilizing solution $(0.1 \%$ triton- $0.1 \%$ sodium citrate in PBS). Cells were then blocked for $1 \mathrm{~h}$ in PBS-0.1\% triton-5\% horse serum (Thermo Fisher Scientific) and incubated overnight at $4{ }^{\circ} \mathrm{C}$ with the following primary antibodies: polyclonal goat anti-human OTX2, 1/500, R\&D; monoclonal rat anti-mouse Zonula Occludens(ZO)-1, 1/200, Millipore and rabbit anti-human hematopoietic transcription factor PU-1, 1/200 diluted in PBS triton $0.1 \%$ and $1 \%$ horse serum. Alexa fluor 594 phalloidin (Invitrogen) was used for F-actin staining. Secondary antibodies produced in donkey were used at room temperature for $1 \mathrm{~h}$ (AlexaFluor $488 \mathrm{~nm}$ and $647 \mathrm{~nm}, 1 / 500$, Thermo Fisher Scientific), and nuclei were counterstained with Hoechst (1/1000, SigmaAldrich). Cells were washed twice in PBS and observed under fluorescence microscope (Arrayscan VTI HCS Reader, Thermo Fisher Scientific). Twenty-five fields per well were analyzed and recorded using the Arrayscan software (HCS iDev Cell Analysis Software, Thermo Fisher Scientific).

\section{Measurement of transepithelial resistance}

Transepithelial resistance of RPE cells grown on transwell inserts was measured daily using a WPI EVOM (World Precision Instruments, Sarasota, FL, USA) device. All measurements were performed in the cell culture hood within $5 \mathrm{~min}$ of removal from the incubator. For TER measurement purposes, the measuring probe was immersed beforehand in $100 \%$ ethanol for $15 \mathrm{~min}$ then incubated in culture medium for two additional hours. Net TER was obtained by subtracting the value of a trans-well filter without cells from that of cellcontaining filters. The TER per unit area $\left(\Omega \mathrm{cm}^{2}\right)$ was obtained by multiplying the raw TER value by the surface of trans-well inserts $\left(0.33 \mathrm{~cm}^{2}\right)$. Before the start of TNF $\alpha$ or anti-TGF $\beta$ treatments, the polarization of RPE cells was determined and confirmed by their high TER $\left(>250 \Omega \mathrm{cm}^{2}\right.$ ). All measurements were expressed as percentage of control.

\section{Phagocytosis assay}

RPE phagocytosis of porcine photoreceptor outer segments (POS) was evaluated according to a previously described protocol [21]. RPE cells were pre-treated in $1 \%$ FCS-1\%PS DMEM in presence of different concentrations of TNF $\alpha$ for 10 days then serum starved $24 \mathrm{~h}$ before evaluating their POS phagocytic capacity (exposure to POS: $3 \mathrm{~h}$ ).

\section{Western blot analysis}

Rhodopsin degradation was analyzed qualitatively in a Western blot (WB) assay. Briefly, RPE cells were previously cultured in $1 \%$ FCS-1\%PS DMEM, with or without different concentrations of TNF $\alpha$ for 10 days, then serum starved before adding the POS (according to a previously described protocol [21]) for 6 and $24 \mathrm{~h}$ respectively. After these incubation periods, supernatants were removed and RIPA buffer was added to RPE cells before performing the WB as previously described [22] using primary anti-Rhodopsin (Merck ref MAB5316) and anti- $\beta$-actin antibodies (Sigma).

\section{Proliferation assay}

DNA replication in RPE cells was assessed in vitro using the Click-it EDU (5-ethynyl-2'-deoxyuridine) Alexa Fluor 594 Imaging kit according to the manufacturer's instructions after fixating the cells in $4 \%$ PFA for $10 \mathrm{~min}$ and preparing them for immunofluorescence microscopy using primary and secondary antibodies as described earlier.

\section{Cell-fusion study}

Freshly collected porcine RPE cells were incubated for $20 \mathrm{~min}$ at $37{ }^{\circ} \mathrm{C}$ in presence of either $5 \mu \mathrm{M}$ CFSE or $1 \mu \mathrm{M}$ Far red CFSE (cell trace, cell proliferation kits, Invitrogen) according to the manufacturer's protocol then kept for $5 \mathrm{~min}$ in 20\%FCS 1\%PS DMEM and centrifuged at $800 \mathrm{rpm}$ for five additional minutes. The cells were then seeded in 16-well glass slide lab-teks (Nunc) at the final concentration of 75,000 cells per well in DMEM-FCS20\%-PS1\% (including 50\% CFSE labeled and 50\% Far red CFSE labeled cells). When confluence was reached, cells were serum starved and treated daily with either DMEM-PS1\% or TNF $\alpha$-enriched medium as previously described. 
Reverse transcription and real-time quantitative polymerase chain reaction

Total RNA from cell cultures was extracted using the Nucleospin RNAII extraction kit according to the manufacturer's protocol (Macherey Nagel). RNA yields were then measured using the NanoDrop ${ }^{\mathrm{TM}} 8000$ spectrophotometer at a $260 \mathrm{~nm}$ wavelength. Overall, RNA concentrations ranged between 40 and $80 \mathrm{ng} / \mu \mathrm{L}$. Reverse transcription into single-strand cDNA was performed using $1 \mu \mathrm{g}$ of total mRNA (pretreated with DNase), oligo-dT, and superscript II reverse transcriptase (Thermo Fisher Scientific). For realtime PCR, $1 / 50$ of cDNA was incubated with the polymerase and appropriate amounts of nucleotides (PowerSYBR Green PCR mix, Applied Biosystems) and PCR was performed using StepOne Plus real-time PCR system (Applied Biosystems). Results were normalized using house-keeping gene RPS26. PCRs were performed in 45 cycles of $15 \mathrm{~s}$ at $95{ }^{\circ} \mathrm{C}, 45 \mathrm{~s}$ at $60{ }^{\circ} \mathrm{C}$. Primers for RT-PCR were purchased from IDT technology. Sequences are as follows: ACTA2 forward 5' -CCAACCGGGAGAAGATGACC- 3'; ACTA 2 reverse 5' - AGAGTCCAGCACAATGCCAG- 3'; CLD19 forward 5'-GCCCTAGCACACCTGTCAAT -3'; CLD19 reverse 5'- ACGTGCAGCAGAGGAACGAG-3'; OCLD forward 5'-ATTTATGACGAGCAGCCCCC-3'; OCLD reverse 5'-GCATAGTCCGAAAGGGGAGG-3'; RPE65 forward 5' - TTCTCTTTTCAGGGCCTCGT-3'; RPE65 reverse 5' - AAAGATGGGTTCGGATGGGT-3'; RPS26 forward 5'-TCGATGCCTATGTGCTTCCC-3'; RPS26 reverse 5'-CAGCACCCGCAGGTCTAAAT-3'; TGF $\beta 1$ forward: TTACAACAGTACCCGCGACC; TGF $\beta 1$ reverse: CCGCTTTCCAGCATTAGCAC; TTR forward 5' - TGGA AGGCACTTGGCATTTC-3'; TTR reverse 5' - GGTGGA GTAAGAGTAGGGGC -3'.

\section{Statistical analyses}

Graph Pad Prism 7 (GraphPad Software) was used for data analysis and graphic representation. All values are reported as mean \pm SEM. Statistical analyses were performed by one-way ANOVA analysis of variance, Student $t$ test or Mann-Whitney test for comparison of mean a

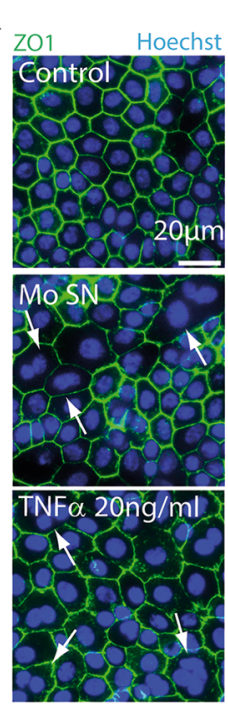

f

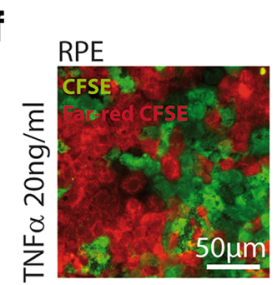

b
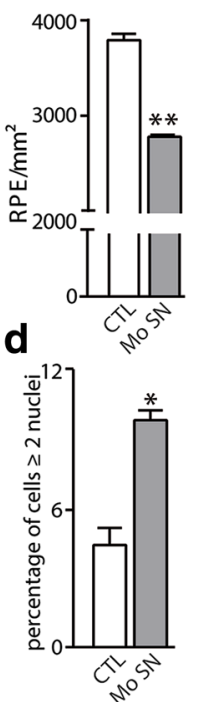

$\mathbf{g}_{70}$

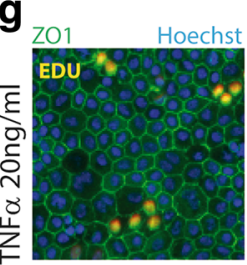

\section{C}
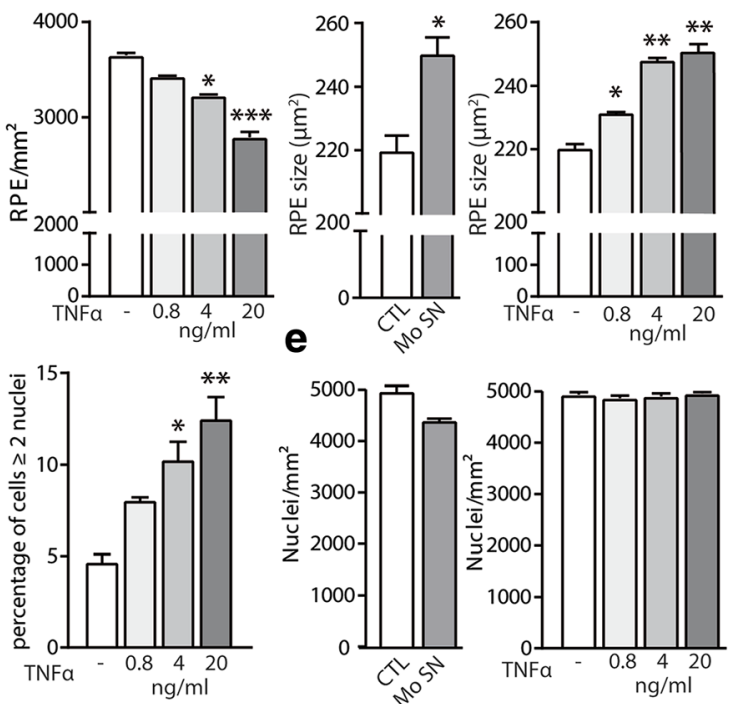

Fig. 1 Chronic exposure to activated monocyte supernatants or TNFa increases the percentage of enlarged, multinucleated RPE cells. Porcine retinal pigment epithelium (RPE) cells were cultured with or without lipopolysaccharide activated monocyte supernatants (Mo SN) or TNFa added daily at different concentrations for a total of 10 days. a Representative pictures of Zonula Occludens (ZO) 1 (green) and Hoechst (blue) immunohistochemistry of control, Mo SN, and TNFa-exposed RPE. Automated quantifications by Arrayscan of $\mathbf{b}$ the number of RPE cells, $\mathbf{c}$ RPE cell size, $\mathbf{d}$ the number of multinucleated RPE cells ( $\geq 2$ nuclei), and e total number of nuclei per $\mathrm{mm}^{2}$ in the indicated different conditions. Representative images of 10-day TNFa-exposed RPE cell-cultures of $\mathbf{f}$ a co-culture of 50\% RPE cells labeled with Carboxyfluorescein succinimidyl ester (CFSE) (green) and 50\% RPE cells labeled with Far-red CFSE (red), and g ZO-1- (green) and Hoechst nuclear- (blue) stain of cultures maintained in the presence of EDU (5-ethynyl-2'-deoxyuridine, red). ( $n=5 /$ group, one way ANOVA or Mann-Whitney, comparison versus control: ${ }^{*} P<0.05$, ${ }^{*} P<0.005^{* * *} P<0.0005$ ) 
values when applicable, followed by Bonferroni post-test. The $p$ values are indicated in the figure legends.

\section{Results}

Chronic exposure to activated monocyte supernatants or TNFa increases the percentage of enlarged, multinucleated RPE cells

In vitro, confluent primary porcine RPE cells form a mosaic of hexagonal and regularly shaped cells with tight junctions that can be visualized by a Zonula Occludens 1 (ZO1) immunohistochemistry (Fig. 1a). Ten daily repeated exposures to lipopolysaccharide (LPS)-stimulated Mo supernatant (Mo SN) or daily exogenously added TNF $\alpha$ did not induce RPE cell monolayer defects but led to enlarged and irregularly shaped RPE cells (Fig. 1a). Automated quantification of the number of ZO1-demarcated RPE cells using an Arrayscan revealed that Mo SN exposure decreased the cell number (Fig. 1b) in parallel to an increase of average RPE cell size (Fig. 1c). TNFa stimulation was sufficient to induce a similar effect in a dose-dependent manner (Fig. 1b, c). The increase in RPE cell size was associated with a significant increase of the percentage of polynucleated cells in both conditions (Fig. 1a arrows and Fig. 1d) that led to a complete compensation of the overall density of nuclei per surface (Fig. 1e). Ten-day culture of 50\% CFSE-stained and 50\% CFSE far red-stained RPE cells showed no red/green double-labeled cells in TNFo-exposed cell-cultures, excluding cell fusion as a mechanism of polynucleation in this condition (Fig. 1f). Addition and revelation of the traceable nucleotide 5-ethynyl-2'-deoxyuridine (EDU) to the TNFoexposed cell-cultures showed that nuclei of multinucleated RPE cells were EDU positive (Fig. 1g), demonstrating that polynucleation was due to nuclei replication and failed cytokinesis as previously shown in aged mice in vivo [3].

In conclusion, 10-day exposure to Mo SN or TNF $\alpha$ alone provided a sufficient stimulus to induce an increase in the percentage of enlarged, multinucleated RPE cells similar to what is observed in vivo with age $[3,4]$.

\section{Chronic TNFa decreases visual cycle gene expression in the RPE}

Transthyretin (TTR), a retinol carrier, and RPE65 that is responsible for the conversion of all-trans-retinyl esters to 11-cis-retinol are strongly expressed by the RPE and essential parts of the visual cycle. We have previously shown that TNF $\alpha$ acutely reduces the expression of TTR and orthodenticle homeobox 2 (OTX2) that regulates its expression [22]. Here, repeated stimulation of primary RPE by TNF $\alpha$ diminished the intensity of OTX2-staining measured with an Arrayscan similar to 10 repeated Mo SN exposures (Fig. 2a). Chronic TNFo exposure also significantly reduced TTR transcription, measured by RT-qPCR, at higher concentrations (Fig. 2b). Additionally, we noticed a dosedependent decrease of RPE65 transcription (Fig. 2c).

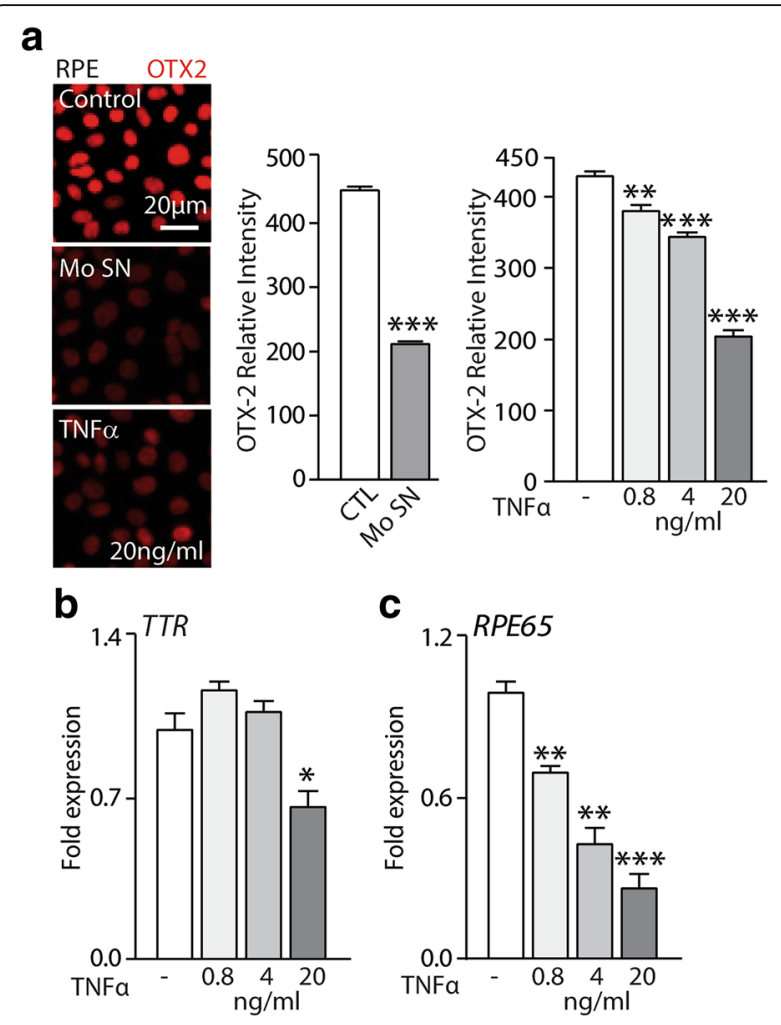

Fig. 2 Chronic exposure to activated monocyte supernatants or TNFa decreases visual cycle gene expression in the RPE. a Representative pictures of RPE staining with OTX2 (Orthodenticle homeobox 2, red) antibody and Arrayscan fluorescence intensity quantification of OTX-2 staining after 10 days of culture with or without lipopolysaccharideactivated monocyte supernatants (Mo SN) or TNFa added daily at different concentrations. b TTR (transthyretin) and c RPE65 (retinal pigment epithelium specific $65 \mathrm{kDa}$ protein)-mRNA expression normalized with \$26 expression quantified by RT-qPCR of 10-day RPE culture exposed to the indicated TNFa concentrations ( $n=5 /$ group one-way ANOVA or MannWhitney, comparison versus control ${ }^{*} P<0.05,{ }^{* *} P<0.005$, ${ }^{* * *} P<0.0005$ )

In summary, we demonstrated that chronic exposure to Mo SN and TNFo downregulated the expression of major RPE visual cycle genes.

\section{Chronic exposure to TNFa disrupts RPE barrier properties}

Physiologically, individual RPE cells are joint by tight junctions (TJ) to form an ion-impermeable epithelium that constitutes the outer blood-retinal barrier from the fenestrated vessels of the choroid. In vitro, this RPE function is reflected by a high transepithelial electrical resistance (TER) [23]. Repeated TNF $\alpha$ exposure of confluent primary RPE cells grown in trans-wells significantly diminished the TER in a dose- and time-dependent manner starting at day 3 (Fig. 3a). Immunohistochemistry of 10-day TNF $\alpha$ exposed RPE cells using ZO1 antibody and phalloidin that binds to $\mathrm{F}$ actin filaments showed a loss of the preferential location to cell borders of both proteins in the 4 and $20 \mathrm{ng} / \mathrm{ml}$ groups (Fig. 3b). Both groups also displayed a 
a

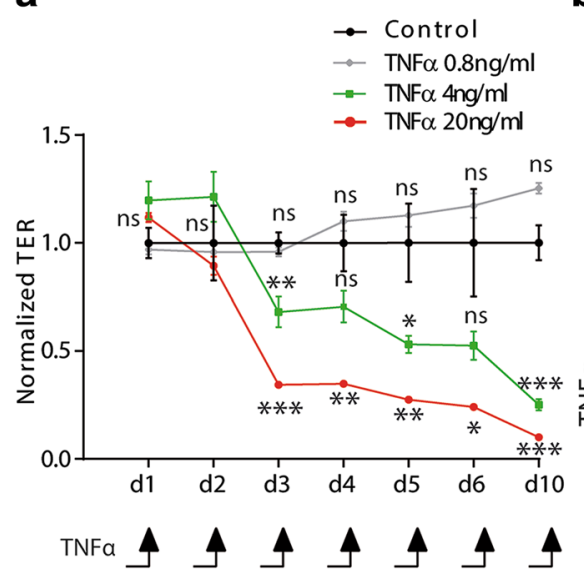

C OCLD

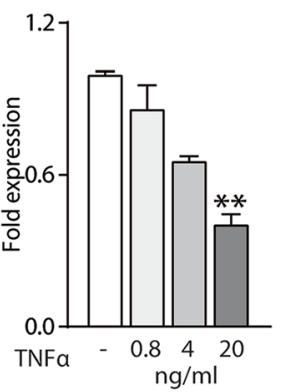

b RPE

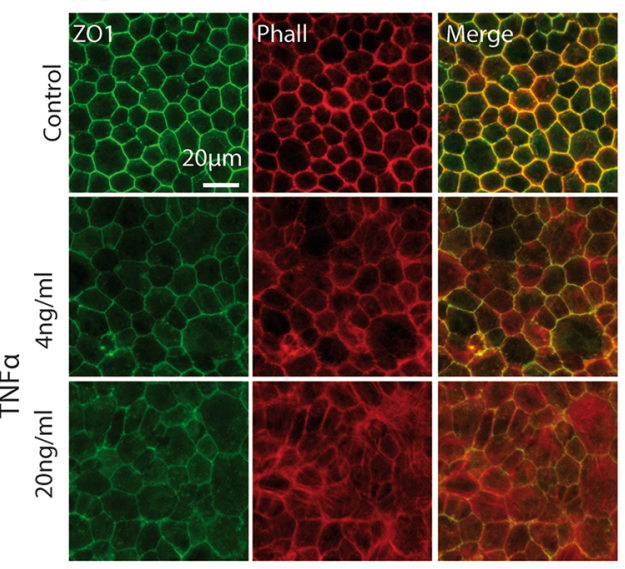

$\mathbf{e}$

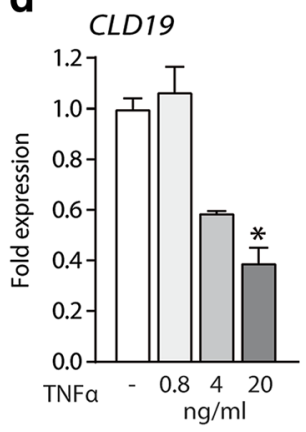

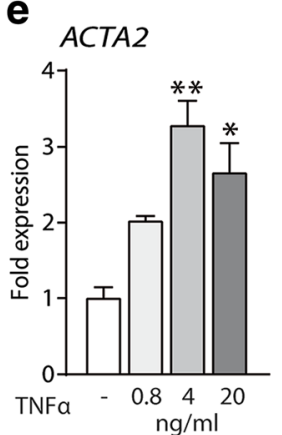

Fig. 3 Chronic exposure to TNFa disrupts RPE barrier properties. a Measurements of RPE transepithelial electric resistance (TER) measured daily in a 10-day trans-well culture with or without different concentrations of TNFa $(n=3 /$ group, one-way ANOVA, versus control for each time point $* P$ $\left.<0.05,{ }^{* *} P<0.005,{ }^{* *} P<0.0005\right)$. b Representative pictures of RPE staining with Zonula occludens(ZO) 1 (green) and F actin (Phalloidin, red). c-e Relative expression of OCLD (Occludin), CLD19 (Claudin-19), and ACTA2 (Smooth muscle alpha (a)-2 actin) mRNA expression normalized with S26 quantified by RT-qPCR of 10-day RPE culture with or without the indicated TNFa concentrations ( $n=5 /$ group one-way ANOVA, versus control * $P$ $\left.<0.05,{ }^{* *} P<0.005,{ }^{* * *} P<0.0005\right)$

significantly inhibited transcription of TJ components occludin and claudin 19 by RT-qPCR at day 10 (Fig. 3c, d). On the contrary, repeated exposure to even $0.8 \mathrm{ng} / \mathrm{ml}$ of TNF $\alpha$ increased the transcription of smooth muscle alpha (a)-2 actin (ACTA2), a major constituent of the contractile fibers that are classically expressed in myofibroblasts and smooth muscle cells (Fig. 3e).

Taken together, our data shows that repeated exposure to TNF $\alpha$ induces profound changes in cytoskeleton and TJ component expression and leads to the loss of the physiological TER that characterizes primary RPE cells.

\section{Chronic exposure to TNFa does not alter the} phagocytosis and degradation of rod outer segments

Phagocytosis of shed photoreceptor outer segments (POS) and recycling is another important role of RPE cells. To examine the effects of chronic TNF $\alpha$ exposure on RPE phagocytic function, we incubated FITC-labeled POS with control and 10-day TNF $\alpha$ pre-treated RPE cells as previously described [21]. After $3 \mathrm{~h}$ of incubation with POS, we noted a tendency to increased POS binding by RPE cells (Fig. 4a), but no difference in the amount of internalized POS between the groups (Fig. 4b). Furthermore, Western blots of rhodopsin of the same protein amount from control and TNF $\alpha$ pre-treated RPE cells, exposed to POS for 6 and $24 \mathrm{~h}$, clearly demonstrated POS degradation after $24 \mathrm{~h}$ in both control and TNF $\alpha$ pre-treated cells (Fig. 4c). If normalized to actin $\beta$ levels, which increased dose dependently with TNF $\alpha$ levels, rhodopsin remnants at $24 \mathrm{~h}$ in TNF $\alpha$ pre-treated cells would seem to be even lower than in the control group (Fig. 4c).

Together, these experiments suggest that chronic TNF $\alpha$ exposure does not impede the RPE's capacity to phagocytize and digest POS.

\section{Chronic exposure to TNFa impairs the immunosuppressive RPE cell function}

The subretinal space is an immunosuppressive environment, mediated in part by immunosuppressive signals of the RPE [2]. In vitro, we have recently shown that 


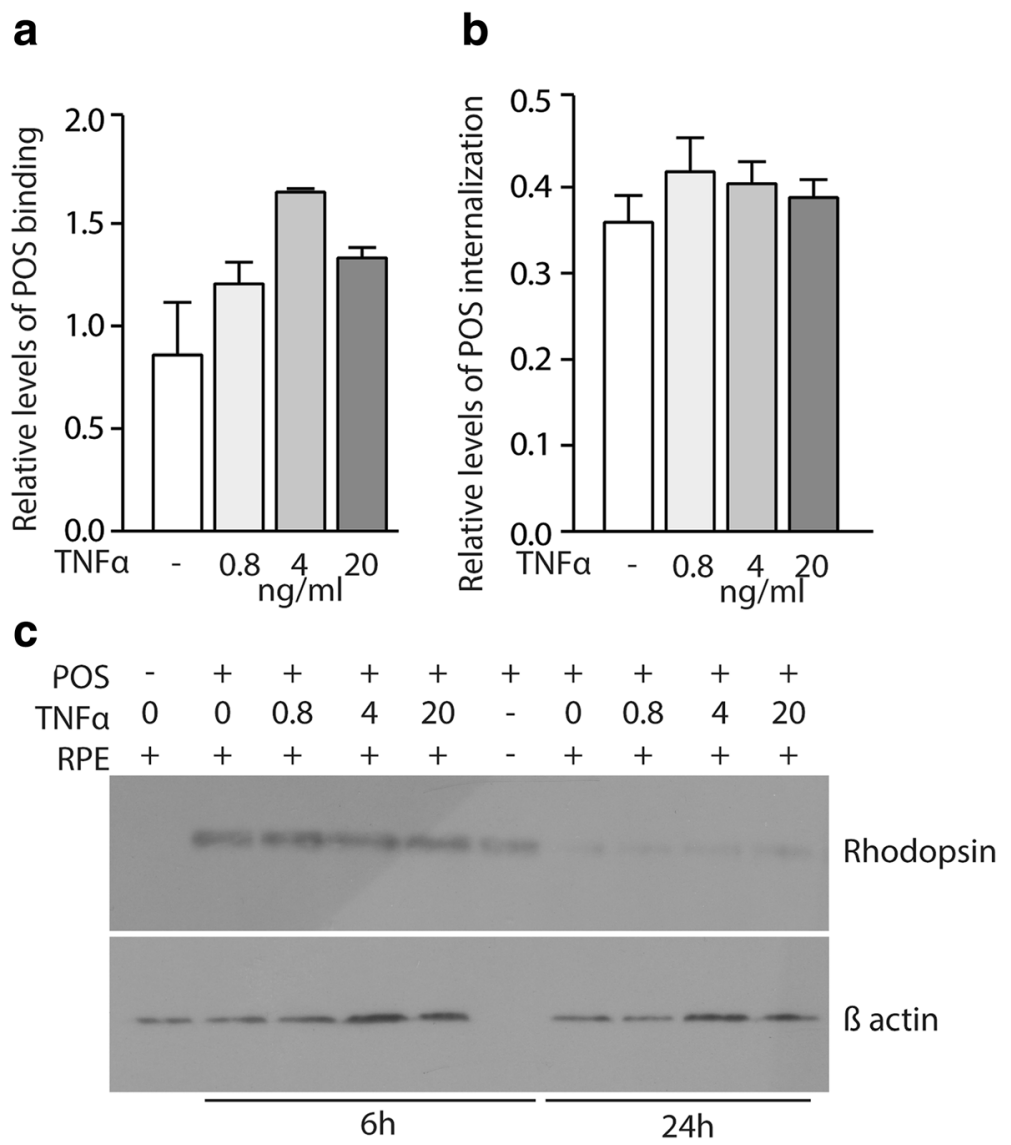

Fig. 4 Chronic exposure to TNFa does not alter the phagocytic and rhodopsin degradation capabilities of RPE cells. a Quantification of relative levels of photoreceptor outer segment (POS) binding and $\mathbf{b}$ POS internalization by RPE cells in the indicated culture conditions ( $n=5 / \mathrm{group}$ one-way ANOVA, versus control, non-significant differences). c Western blot analysis of Rhodopsin and $\beta$-actin protein after 6 and 24 h of culture of primary porcine RPE cells (pretreated with 10-day TNF-a at 0.8, 4, and $20 \mathrm{ng} / \mathrm{mL}$ ) and photoreceptor outer segments (POS)

primary RPE cells rapidly induce the elimination of nonactivated monocytes (Mo) in a cell-contact dependent manner [22]. To test whether chronic TNF $\alpha$ exposure altered the RPE's capacity to eliminate Mo, we incubated equal numbers of peripheral blood Mo with control and 10-day TNF $\alpha$ pre-treated RPE cells for $24 \mathrm{~h}$ and stained the co-culture with an anti-PU1-antibody that specifically recognizes Mo, which express this transcription factor, contrary to RPE cells (Fig. 5a). Arrayscan quantification of the number of $\mathrm{PU} 1^{+} \mathrm{Mo}$ demonstrates up to a $500 \%$ increase in Mo numbers in the co-culture conditions, where the RPE was pre-incubated repeatedly with TNFa (Fig. 5b).

Taken together, our data demonstrates that chronic exposure of RPE to TNF $\alpha$ severely diminishes its immunosuppressive capacities.

\section{TNFa-induced TGF- $ß$ expression mediates the RPE} polynucleation and impairement of barrier properties TNF $\alpha$ induces the expression and DNA binding of AP-1 (activator protein-1) resulting in increased transcription of the TGF- $\beta 1$ gene in lung fibroblasts [24]. Furthermore, TGF- $\beta 1$ has been shown to mediate fibrosis in the lung and to induce RPE dedifferentiation in the context of retinal detachment and proliferative vitreoretinopathy [25].

RT-qPCR analysis showed that repeated TNF $\alpha$ exposure induced a strong increase of TGF $\beta 1$ transcription at 2 days that remained increased after 10 days of culture (Fig. 6a). Interestingly, the coadministration of TGF $\beta$-receptor inhibitor (500 nM) to the RPE culture inhibited the TNF $\alpha$-induced polynucleation described in Fig. 1 (Fig. 6b). Similarly, TGF $\beta$-receptor inhibitor prevented the TNF $\alpha$ induced dislocation of the RPE junction protein $\mathrm{ZO} 1$ and F-actin (Fig. 6c) and partially restored the TER (Fig. 6d). On the other hand, the inhibition of TGF $\beta$ signaling did not prevent the TNF $\alpha$-induced decrease in OTX2 expression (data not shown).

Taken together, these data strongly suggest that some, but not all, of the TNF $\alpha$-induced RPE dysfunction is mediated by increased TGF- $\beta$ signaling that has previously been shown to perturb certain RPE functions. 


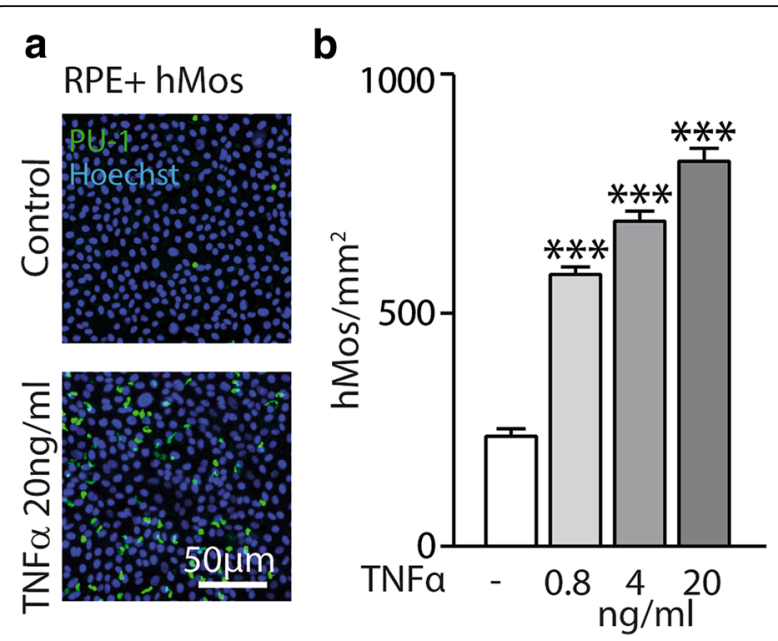

Fig. 5 Chronic exposure to TNFa impairs the immunosuppressive function of RPE cells. a Representative pictures of human monocytes (hMos, human hematopoietic transcription factor (PU1) staining, green) and RPE nuclei (Hoechst, blue) and $\mathbf{b}$ automated quantification of the number of human monocytes in a 24-h co-culture with RPE cells with or without previous 10-day pre-treatment with TNFa (at 0.8, 4, and $20 \mathrm{ng} / \mathrm{ml})\left(n=5 /\right.$ group, one way ANOVA versus control $\left.{ }^{* * *} P<0.0005\right)$

\section{Discussion}

We and others previously showed that Mфs accumulate on the apical side of the RPE adjacent to atrophic zones that define GA, around choroidal neovascularizations and around large drusen in intermediate AMD [2, 8-12, 26]. The RPE is therefore likely chronically exposed to M $\phi-$ derived cytokines that might alter its function. One of the key cytokines of potential interest is TNF $\alpha$, which increases with age in plasma and Mфs $[14,15,17,18]$ and is increased in CFH AMD-risk variant carriers [16]. We recently demonstrated that M $\phi$-derived TNF- $\alpha$ acutely downregulates the transcription factor orthodenticle homeobox 2 (OTX2) that is constitutively expressed in RPE cells, where it regulates a number of essential RPE genes [22] and TNF- $\alpha$ exposure has been shown to acutely diminish RPE barrier functions [23]. We here exposed primary RPE cells to 10 daily repeated TNF $\alpha$ stimulations to mimic the exposure of in vivo chronic inflammatory conditions and evaluated its impact on RPE morphology and its main distinctive functions: visual cycle gene expression, tight-junction organization and transepithelial resistance, POS phagocytosis and ROS degradation, and its immunosuppressive capacities. We exposed the RPE to a range of TNF $\alpha$ concentrations from 0.8 to $20 \mathrm{ng} / \mathrm{ml}$, which we administered daily, as TNF $\alpha$ has been reported to have a short half-life of around $15 \mathrm{~min}$ [27] and compared some of the findings to the exposure of supernatants from $24 \mathrm{~h}$ LPSactivated monocytes (Mo SN).

First, using ZO1/nuclear Hoechst immunohistochemistry, we observed that after 10 days of exposure to Mo $\mathrm{SN}$, the RPE remained confluent. However, the number of RPE cells had decreased significantly, which was compensated by an increased, albeit irregularly shaped, cellsize. In parallel, the percentage of polynucleated cells was significantly increased compared to controls. TNF $\alpha$ exposure was sufficient to induce very similar morphological differences in a dose-dependent manner. TNF $\alpha$ exposure of cultures using a mixture of red- and greenlabeled RPE cells and EdU incorporation assays also revealed that the polynucleated cells were not the result of cell fusion but the consequence of nucleic replication in the polynucleated cells. Interestingly, the nucleic replication exactly compensated for the cells lost during the 10-day TNF $\alpha$ and Mo SN exposure, suggesting that the number of nuclei per RPE was finely regulated by their cell size. Interestingly a similar mechanism is also observed in vivo with age [3] that is associated with increased TNF $\alpha$ levels $[14,15,17,18]$ and in the vicinity of large drusen [5]. Although these differences were observed in the $0.8 \mathrm{ng} / \mathrm{ml}$, and $4 \mathrm{ng} / \mathrm{ml}$ group, they were most prominent in the $20 \mathrm{ng} / \mathrm{ml}$ group and similar to Mo SN exposure.

We next evaluated if chronic TNF $\alpha$ exposure influenced the expression of visual cycle genes. We have previously shown that TNF $\alpha$ acutely downregulates the expression of the transcription factor OTX2 that regulates a number of RPE-specific genes, notably TTR that is involved in the visual cycle [22]. Similarly, chronic TNF $\alpha$ exposure inhibited OTX2 expression, alike to Mo SN exposure, evaluated by immunohistochemistry and fluorescence quantification. It not only diminished the transcription of TTR but also induced a strong and dose dependent reduction in the expression of RPE65 that is not controlled by OTX2. Our observations in primary RPE cells thereby reproduce similar findings in an RPE cell line [28]. Together these results confirm our previous findings and suggest that chronic TNF $\alpha$ exposure reduces the expression of several RPE-specific genes that are implicated in the visual cycle. Dysfunction of the visual cycle leads to an increase of recovery time in the scotopic ERG after bleach, as it takes more time to reproduce 11-cis-retinal. Interestingly, patients with large drusen that are associated with polynucleated RPE cells [5] also present a marked increase in recovery time after bleach $[6,7]$, which could be due to exposure to TNF $\alpha$ secreted by the associated chronic infiltrate.

Physiologically, individual RPE cells are joint by TJs and the confluent RPE forms an ion-impermeable epithelium that constitutes the outer blood-retinal barrier from the fenestrated vessels of the choroid. We here show that repeated TNF- $\alpha$ exposure of primary RPE induces a dosedependent loss of the TER, a functional measure of its barrier function, at $4 \mathrm{ng} / \mathrm{ml}$ and $20 \mathrm{ng} / \mathrm{ml}$, that worsens with each TNF- $\alpha$ application. Both, $4 \mathrm{ng} / \mathrm{ml}$ and $20 \mathrm{ng} / \mathrm{ml}$ TNF- $\alpha$-exposed RPE cells also displayed a loss of the 

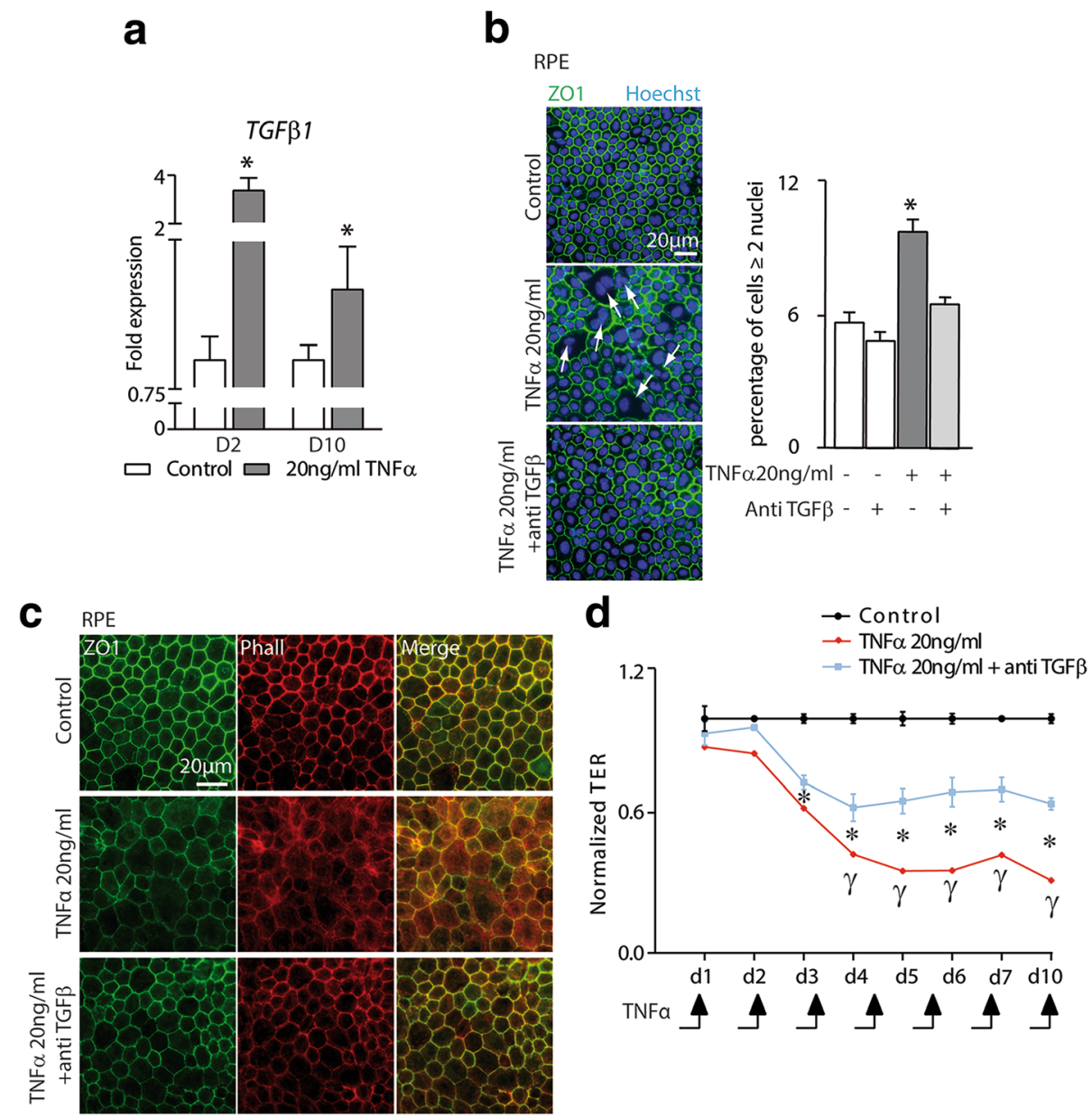

Fig. 6 TNFa-induced TGF- $\beta$ expression mediates the RPE polynucleation and impairment of barrier properties. a TGF $\beta$ 1-mRNA expression normalized with S26 expression quantified by RT-qPCR of 2, and 10-day RPE culture exposed to $20 \mathrm{ng} / \mathrm{ml}$ of TNFa. b Representative pictures of Zonula Occludens (ZO) 1 (green) and Hoechst (blue) immunohistochemistry of control, TNFa(20 ng/ml), and TNFa(20 ng/ml) + antiTGF $\beta$ (500 nM)-exposed RPE. Quantifications of the number of multinucleated RPE cells ( $\geq 2$ nuclei) in the indicated conditions. (a, $\mathbf{b}, n=5 /$ group, one-way ANOVA or Mann-Whitney versus control $\left.{ }^{*} P<0.05,{ }^{* *} P<0.005,{ }^{* * *} P<0.0005\right)$. c Representative pictures of RPE staining with Zonula occludens (ZO) 1 (green) and F actin (Phalloidin, red) after 10 days of culture in the indicated conditions. $\mathbf{d}$ RPE transepithelial electric resistance (TER) measured daily in a 10-day trans-well culture with or without TNFa $(20 \mathrm{ng} / \mathrm{ml})$ and TNFa $(20 \mathrm{ng} / \mathrm{ml})+$ antiTGF- $\beta(500 \mathrm{nM})\left(n=3 / \mathrm{group},{ }^{*} P<0.05\right.$ one-way ANOVA of control versus TNFa and TNFa + antiTGF- $\beta$ for each time point, $\Upsilon P<0.05$ Mann-Whitney of TNFa + antiTGF- $\beta$ versus TNFa for each time point)

preferential location of the TJ protein ZO1 and F actin filaments to cell borders, diminished transcription of the TJ components occludin and claudin19, and increased expression of smooth muscle alpha ( $\alpha$ )-2 actin (ACTA2), in accordance with altered barrier functions. A similar decrease of TER has previously been described after $24 \mathrm{~h}$ of a single stimulation with TNF- $\alpha$ at 10 to $50 \mathrm{ng} / \mathrm{ml}$ [23], which was not observed after a stimulation with $100 \mathrm{pg} /$ $\mathrm{ml}$ [29]. Taken together, our data and the data from the literature suggest that exposure to TNF- $\alpha$, and in particular repeated exposure, severely impedes RPE barrier functions.

We next evaluated if chronic TNFo-exposure affected the RPE's capacity to phagocyte and digest POS, an important function for their recycling and renewal.
Interestingly, the phagocytosis assay revealed a tendency of increased POS binding, little differences in POS uptake, and no differences in rhodopsin degradation, the major protein of POS. These results suggest that chronic TNF $\alpha$ exposure has little influence on RPE phagocytosis, contrary to the other examined functions.

Last but not least, we examined the immunosuppressive capacity of TNF $\alpha$ pre-exposed RPE by incubating the cells with monocytes after their 10-day cytokine exposure. While monocytes were quickly eliminated by the contact with control RPE for $24 \mathrm{~h}$, as previously described [22], we observed a very significant dose-dependent increase in monocyte survival of the co-culture in TNF $\alpha$-exposed $\mathrm{RPE}$, suggesting that their capacity to induce monocyte death was severely reduced. 
TNF $\alpha$ induces the expression and DNA binding of AP-1 resulting in increased transcription of the TGF- $\beta 1$ gene in lung fibroblasts [24]. Our results show that it similarly induces TGF- $\beta 1$ in RPE cells. Interestingly, pharmacological inhibition of TGF- $\beta$ signaling prevented the TNF $\alpha-$ induced increase in polynucleated RPE cells and reduced the decline of the TER but did not restore OTX2 expression levels. These results suggest that some, but not all, of the TNF $\alpha$-induced effects are mediated by TGF- $\beta 1$.

\section{Conclusions}

Taken together our results indicate that chronic TNF $\alpha$ exposure is sufficient to alter RPE morphology and impede three out of four cardinal features that define the differentiated RPE. These alterations bare striking similarities to RPE alterations with age and dysfunctions observed in AMD, which are associated with macrophage infiltration [2] and likely chronic TNF $\alpha$ exposure [14-19]. Our results therefore suggest that strategies inhibiting TNF $\alpha$ signaling in RPE cells might help preserve its essential functions and slow degeneration in late AMD.

\begin{abstract}
Abbreviations
M4: Macrophage; ACTA2: Smooth muscle alpha (a)-2 actin; AMD: Age-related macular degeneration; $\mathrm{CFH}$ : Complement factor $\mathrm{H}$; CFSE: Carboxyfluorescein succinimidyl ester; CLD19: Claudin 19; CNV: Choroidal neovascularization; DMEM: Dulbecco Modified Eagle Medium; EDTA: Ethylenediaminetetraacetic acid; EDU: 5-ethynyl-2'-deoxyuridine; ERG: Electroretinogram; FCS: Fetal calf serum; GA: Geographic atrophy; Mo: Monocyte; OCLD: Occludin; OTX2: Orthodenticle homeobox 2; PBS: Phosphate buffered saline; PFA: Paraformaldehyde; POS: Photoreceptor outer segment; PS: Penicillin streptomycin; qPCR: Quantitative polymerase chain reaction; ROS: Rod outer segments; RPE: Retinal pigment epithelium; RPE65: Retinal pigment epithelium specific 65 kDa protein; RT: Reverse transcription; TER: Trans-epithelial resistance; TGF $\beta$ : Transforming growth factor beta; TJ: Tight junction; TNFa: Tumor necrosis factor alpha; TTR: Transthyretin; ZO1: Zonula occludens 1
\end{abstract}

\section{Acknowledgements}

We wish to thank A. Potey and M. Lechuga from the high-throughput screening platform, supported by Department de Paris and by Region lle de France, and Olivier Goureau and Tristan Bodier for invaluable intellectual and technical assistance.

\section{Funding}

This work was supported by grants from INSERM, ANR Geno 2009 (R09099DS), ANR MACLEAR (ANR-15-CE14-0015-01), LABEX LIFESENSES [ANR-10-LABX-65] supported by the ANR (Investissements d'Avenir programme [ANR-11-IDEX0004-02]), and a generous donation by Doris and Michael Bunte.

\section{Availability of data and materials}

All data generated or analyzed during this study are included in this published article. The datasets used and/or analyzed during the current study are also available from the corresponding author on reasonable request.

\section{Authors' contributions}

ST made substantial contributions to acquisition of data, analysis and interpretation of data; drafted the manuscript; gave final approval of the version to be published; and agreed to be accountable for all aspects of the work. FB, SA, HCM, LV, EFN, SR, VF, TM, JS, BB, and XG made substantial contributions to analysis and interpretation of data; revised the manuscript critically for important intellectual content; gave final approval of the version to be published; and agreed to be accountable for all aspects of the work. FS made substantial contributions to conception and design, analysis and interpretation of data; drafted the manuscript; gave final approval of the version to be published; and agreed to be accountable for all aspects of the work.

\section{Ethics approval and consent to participate}

The protocol for human blood sampling and monocyte preparation was approved by the Direction Générale pour la Recherche et I'Innovation of the Ministère de l'Enseignement et de la Recherche (Dossier $n^{\circ 14.007)}$ and by the Commission Nationale de I'Informatique et des Libertés (N/Ref.: IFP/ MKE/AR144088)

\section{Consent for publication}

Not applicable.

\section{Competing interests}

The authors declare that they have no competing interests.

\section{Publisher's Note}

Springer Nature remains neutral with regard to jurisdictional claims in published maps and institutional affiliations.

\section{Author details}

'Sorbonne Université, INSERM, CNRS, Institut de la Vision, 17 rue Moreau, F-75012 Paris, France. '2Department of Ophthalmology, Reference Center in Rare diseases, DHU Sight Restore, Hôpital Pitié Salpêtrière, University Paris VI, 47-83 Boulevard de l'Hôpital, 75013 Paris, France. ${ }^{3} \mathrm{CHNO}$ des Quinze-Vingts, DHU Sight Restore, INSERM-DGOS CIC 1423, 28 rue de Charenton, F-75012 Paris, France.

Received: 13 September 2017 Accepted: 25 February 2018 Published online: 16 March 2018

\section{References}

1. Strauss O. The retinal pigment epithelium. Physiol Rev. 2005;85:845-81.

2. Guillonneau X, Eandi CM, Paques M, Sahel J-A, Sapieha P, Sennlaub F. On phagocytes and macular degeneration. Prog Retin Eye Res. 2017:61:98-128.

3. Chen M, Rajapakse D, Fraczek M, Luo C, Forrester JV, Xu H. Retinal pigment epithelial cell multinucleation in the aging eye-a mechanism to repair damage and maintain homoeostasis. Aging Cell. 2016;15:436-45.

4. Starnes AC, Huisingh C, McGwin G, Sloan KR, Ablonczy Z, Smith RT, et al. Multinucleate retinal pigment epithelium cells of the human macula exhibit a characteristic and highly specific distribution. Vis Neurosci. 2016;33:e001.

5. Al-Hussaini H, Schneiders M, Lundh P, Jeffery G. Drusen are associated with local and distant disruptions to human retinal pigment epithelium cells. Exp Eye Res. 2009:88:610-2.

6. Flamendorf J, Agrón E, Wong WT, Thompson D, Wiley HE, Doss EL, et al. Impairments in dark adaptation are associated with age-related macular degeneration severity and reticular pseudodrusen. Ophthalmology. 2015; 122:2053-62.

7. Owsley C, Jackson GR, Cideciyan AV, Huang Y, Fine SL, Ho AC, et al. Psychophysical evidence for rod vulnerability in age-related macular degeneration. Invest Ophthalmol Vis Sci. 2000;41:267-73.

8. Combadière C, Feumi C, Raoul W, Keller N, Rodéro M, Pézard A, et al. CX3CR1-dependent subretinal microglia cell accumulation is associated with cardinal features of age-related macular degeneration. J Clin Invest. 2007; 117:2920-8.

9. Lad EM, Cousins SW, Van Arnam JS, Proia AD. Abundance of infiltrating CD163+ cells in the retina of postmortem eyes with dry and neovascular age-related macular degeneration. Graefes Arch Clin Exp Ophthalmol. 2015; 253:1941-5.

10. Levy O, Calippe B, Lavalette S, Hu SJ, Raoul W, Dominguez E, et al. Apolipoprotein E promotes subretinal mononuclear phagocyte survival and chronic inflammation in age-related macular degeneration. EMBO Mol Med. 2015;7:211-26.

11. Sennlaub F, Auvynet C, Calippe B, Lavalette S, Poupel L, Hu SJ, et al. CCR2(+ ) monocytes infiltrate atrophic lesions in age-related macular disease and mediate photoreceptor degeneration in experimental subretinal inflammation in Cx3cr1 deficient mice. EMBO Mol Med. 2013;5:1775-93.

12. Gupta N, Brown KE, Milam AH. Activated microglia in human retinitis pigmentosa, late-onset retinal degeneration, and age-related macular degeneration. Exp Eye Res. 2003;76:463-71.

13. Sedger LM, McDermott MF. TNF and TNF-receptors: from mediators of cell death and inflammation to therapeutic giants-past, present and future. Cytokine Growth Factor Rev. 2014;25:453-72. 
14. Bruunsgaard H, Skinhøj P, Pedersen AN, Schroll M, Pedersen BK. Ageing, tumour necrosis factor-alpha (TNF-alpha) and atherosclerosis. Clin Exp Immunol. 2000;121:255-60.

15. Paolisso G, Rizzo MR, Mazziotti G, Tagliamonte MR, Gambardella A, Rotondi $M$, et al. Advancing age and insulin resistance: role of plasma tumor necrosis factor-alpha. Am J Phys. 1998;275:E294-9.

16. Cao S, Ko A, Partanen M, Pakzad-Vaezi K, Merkur AB, Albiani DA, et al. Relationship between systemic cytokines and complement factor $\mathrm{H} Y 402 \mathrm{H}$ polymorphism in patients with dry age-related macular degeneration. Am J Ophthalmol. 2013;156:1176-83.

17. Agrawal A, Agrawal S, Cao J-N, Su H, Osann K, Gupta S. Altered innate immune functioning of dendritic cells in elderly humans: a role of phosphoinositide 3-kinase-signaling pathway. J Immunol. 2007;178:6912-22.

18. Alvarez-Rodríguez L, López-Hoyos M, Muñoz-Cacho P, Martínez-Taboada VM. Aging is associated with circulating cytokine dysregulation. Cell Immunol. 2012;273:124-32.

19. Cousins SW, Espinosa-Heidmann DG, Csaky KG. Monocyte activation in patients with age-related macular degeneration: a biomarker of risk for choroidal neovascularization? Arch Ophthalmol. 2004;122:1013-8.

20. Arnault E, Barrau C, Nanteau C, Gondouin P, Bigot K, Viénot F, et al. Phototoxic action spectrum on a retinal pigment epithelium model of agerelated macular degeneration exposed to sunlight normalized conditions. PLoS One. 2013;8:e71398.

21. Law A-L, Parinot C, Chatagnon J, Gravez B, Sahel J-A, Bhattacharya SS, et al. Cleavage of Mer tyrosine kinase (MerTK) from the cell surface contributes to the regulation of retinal phagocytosis. J Biol Chem. 2015;290:4941-52.

22. Mathis T, Housset M, Eandi C, Beguier F, Touhami S, Reichman S, et al. Activated monocytes resist elimination by retinal pigment epithelium and downregulate their OTX2 expression via TNF-a. Aging Cell. 2017;16:173-82.

23. Shirasawa M, Sonoda S, Terasaki H, Arimura N, Otsuka H, Yamashita T, et al. TNF-a disrupts morphologic and functional barrier properties of polarized retinal pigment epithelium. Exp Eye Res. 2013;1 10:59-69.

24. Sullivan DE, Ferris $M$, Nguyen $H$, Abboud $E$, Brody AR. TNF-alpha induces TGF-beta1 expression in lung fibroblasts at the transcriptional level via AP-1 activation. J Cell Mol Med. 2009:13:1866-76.

25. Saika S, Kono-Saika S, Tanaka T, Yamanaka O, Ohnishi Y, Sato M, et al. Smad3 is required for dedifferentiation of retinal pigment epithelium following retinal detachment in mice. Lab Investig J Tech Methods Pathol. 2004;84:1245-58.

26. Eandi $\mathrm{CM}$, Charles Messance $\mathrm{H}$, Augustin S, Dominguez E, Lavalette $\mathrm{S}$, Forster $\mathrm{V}$, et al. Subretinal mononuclear phagocytes induce cone segment loss via IL-1ß. elife. 2016;5. https://doi.org/10.7554/eLife.16490.

27 Moritz T, Niederle N, Baumann J, May D, Kurschel E, Osieka R, et al. Phase I study of recombinant human tumor necrosis factor alpha in advanced malignant disease. Cancer Immunol Immunother CII. 1989;29:144-50.

28 Kutty RK, Samuel W, Boyce K, Cherukuri A, Duncan T, Jaworski C, et al. Proinflammatory cytokines decrease the expression of genes critical for RPE function. Mol Vis. 2016;22:1156-68.

29 Zech JC, Pourreau I, Cotinet A, Goureau O, Le Varlet B, de Kozak Y. Effect of cytokines and nitric oxide on tight junctions in cultured rat retinal pigment epithelium. Invest Ophthalmol Vis Sci. 1998;39:1600-8.

\section{Submit your next manuscript to BioMed Central and we will help you at every step:}

- We accept pre-submission inquiries

- Our selector tool helps you to find the most relevant journal

- We provide round the clock customer support

- Convenient online submission

- Thorough peer review

- Inclusion in PubMed and all major indexing services

- Maximum visibility for your research

Submit your manuscript at www.biomedcentral.com/submit
Biomed Central 